

open research
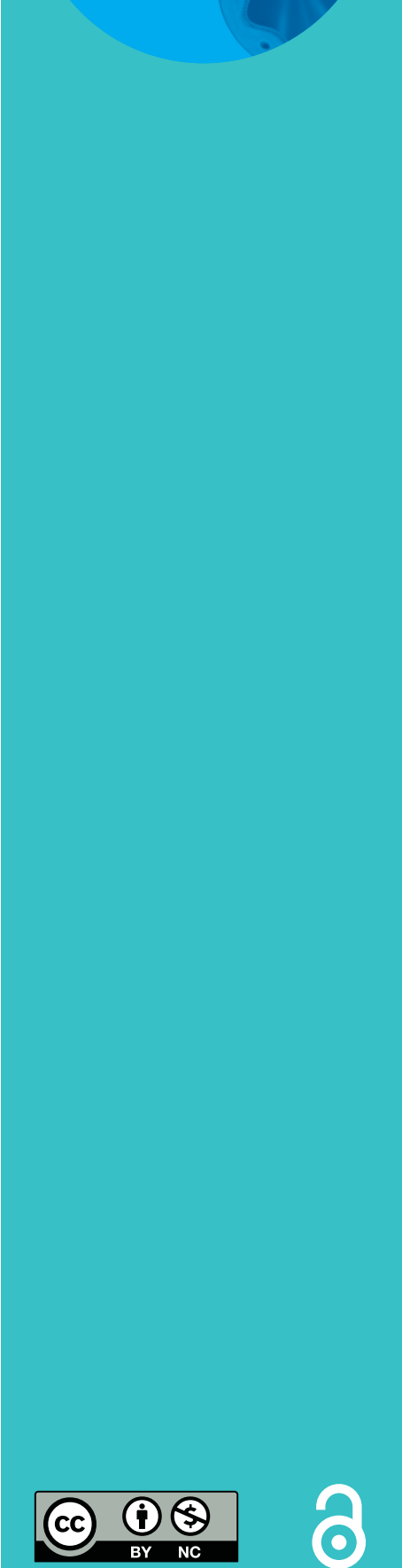

\section{Reproducibility of hypercapnic ventilatory response measurements with steady- state and rebreathing methods}

\author{
Denise C. Mannée ${ }^{1}{ }^{1,2}$, Timon M. Fabius ${ }^{1}$, Michiel Wagenaar ${ }^{1}$, \\ Michiel M.M. Eijsvogel ${ }^{1}$ and Frans H.C. de Jongh ${ }^{1,3}$
}

Affiliations: ${ }^{1}$ Dept of Pulmonology, Medisch Spectrum Twente, Enschede, The Netherlands. ${ }^{2}$ Faculty of Science and Technology, University of Twente, Enschede, The Netherlands. ${ }^{3}$ Engineering Technology Faculty, University of Twente, Enschede, the Netherlands.

Correspondence: Denise C. Mannée, Dept of Pulmonology, Medisch Spectrum Twente, PO Box 50000, Enschede, The Netherlands. E-mail: dcmanneedigmail.com

ABSTRACT In this study, the hypercapnic ventilatory response (HCVR) was measured, defined as the ventilation response to carbon dioxide tension $\left(\mathrm{PCO}_{2}\right)$. We investigated which method, rebreathing or steady-state, is most suitable for measurement of the HCVR in healthy subjects, primarily based on reproducibility. Secondary outcome parameters were subject experience and duration.

20 healthy adults performed a rebreathing and steady-state HCVR measurement on two separate days. Subject experience was assessed using numeric rating scales (NRS). The intraclass correlation coefficient (ICCs) of the sensitivity to carbon dioxide above the ventilatory recruitment threshold and the projected apnoea threshold were calculated to determine the reproducibility of both methods.

The ICCs of sensitivity were 0.89 (rebreathing) and 0.56 (steady-state). The ICCs of the projected apnoea threshold were 0.84 (rebreathing) and 0.25 (steady-state). The steady-state measurement was preferred by 16 out of 20 subjects; the differences in NRS scores were small.

The hypercapnic ventilatory response measured using the rebreathing setup provided reproducible results, while the steady-state method did not. This may be explained by high variability in end-tidal $P_{C_{2}}$. Differences in subject experience between the methods are small.

@ERSpublications

The hypercapnic ventilatory response measured by a rebreathing setup is reproducible, as opposed to a steady-state setup http://ow.ly/yGDg30hStIB

Cite this article as: Mannée DC, Fabius TM, Wagenaar M, et al. Reproducibility of hypercapnic ventilatory response measurements with steady-state and rebreathing methods. ERJ Open Res 2018; 4: 00141-2017 [https://doi.org/10.1183/23120541.00141-2017].

Received: Nov 072017 | Accepted after revision: Jan 112018

Conflict of interest: None declared.

Copyright $\odot$ ERS 2018. This article is open access and distributed under the terms of the Creative Commons Attribution Non-Commercial Licence 4.0. 


\section{Introduction}

The control of breathing is regulated mainly through the central and peripheral chemoreceptors. Central chemoreceptors react to changes in carbon dioxide tension $\left(\mathrm{PCO}_{2}\right)$ and hydrogen concentration. Peripheral chemoreceptors respond to the same stimuli and to low oxygen tension levels [1-4]. Two types of method are known to measure the central chemoresponse to $\mathrm{PCO}_{2}$ : rebreathing and steady-state methods (e.g. Read's rebreathing, Duffin's rebreathing, dynamic end-tidal forcing and prospective targeting) [4]. Under hyperoxic conditions, the peripheral response to $\mathrm{PCO}_{2}$ is diminished and can be neglected. In healthy subjects, the difference between the end-tidal $\mathrm{CO}_{2}$ tension $\left(\mathrm{ETCO}_{2}\right)$ and arterial $\mathrm{PCO}_{2}\left(\mathrm{PaCO}_{2}\right)$ is negligible. Numerous factors (e.g. cerebral blood flow) determine the relationship between $\mathrm{PaCO}_{2}$ and $\mathrm{CO}_{2}$ tension at the central chemoreceptors $\left(P_{\mathrm{CCO}_{2}}\right)$. Nevertheless, $P_{\mathrm{ETCO}}$ is often used as a surrogate for $P_{\mathrm{CCO}_{2}}$, as measurement of $\mathrm{PCCO}_{2}$ itself is too invasive. In both methods the $\mathrm{PETCO}_{2}$ is measured as an input and ventilation as output. In rebreathing methods, $\mathrm{CO}_{2}$ in the exhaled air of a subject is re-used (rebreathed), to increase $\mathrm{PCCO}_{2}$ continuously. In steady-state methods, it is assumed that a time delay exists between $\mathrm{PETCO}_{2}$ and $\mathrm{PCCO}_{2}$. To create an equilibrium between $P_{\mathrm{CCO}_{2}}$ and $\mathrm{PETCO}_{2}, P_{\mathrm{ETCO}}$ is maintained at a constant level for 5-10 min [4]. The chemoresponse function is linearly proportional to $\mathrm{PCO}_{2}$ above the ventilatory recruitment threshold (VRT), and is referred to as the hypercapnic ventilatory response (HCVR), i.e. the change in minute volume induced by chemoreceptors due to changes in $\mathrm{PCO}_{2}$. At $\mathrm{PCO}_{2}$ levels below VRT, chemoreceptors are not sensitive to $\mathrm{PCO}_{2}$ changes, and ventilation is driven by the "wakefulness drive". Theoretically, in sleep the wakefulness drive disappears and the function of the controller becomes completely linear [4]. The $\mathrm{PCO}_{2}$ level where, hypothetically, ventilation ceases, is the apnoea threshold.

Numerous systems are known to measure the ventilatory response via rebreathing or steady-state methods. The systems can be controlled manually or by computer (e.g. dynamic end-tidal forcing systems or prospective targeting with the RespirAct ${ }^{\mathrm{TM}}$ (Thornhill Medical, Toronto, ON, Canada) [4]. None of these systems is accepted as a gold standard for measurement of the ventilatory response.

Therefore, the aim of this study was to investigate which method is most suitable for measurement of the HCVR in healthy subjects, based primarily on reproducibility. Reproducibility of a measurement is important, as low reproducibility implies that the outcome of the measurement can vary over time, so limited conclusions can be based on a single measurement. In various publications variation and/or reproducibility of rebreathing and steady-state tests were investigated [5-8]. However, conclusions in more than half of these studies are based on the reproducibility calculated with results of multiple averaged HCVR measurements. This study focuses on the reproducibility of the measured HCVR with steady-state measurement in comparison to rebreathing measurement in a large population.

Second, both methods are reviewed on subject experience and duration of measurement. In the measurement of the ventilatory response, $\mathrm{P}_{\mathrm{CCO}_{2}}$ typically increases above $7 \mathrm{kPa}[6,9]$. It is known that (transient or mild) hypercapnia can cause a sensation of dyspnoea, sluggishness and headaches [10]. However, no studies reported the burden of the measurements on the subjects.

\section{Methods}

\section{Subjects}

Based on previous studies of similar design, 20 subjects were included [8]. The medical ethics committee of Twente (Enschede, the Netherlands) approved the study, as did the local board of directors. All subjects provided written informed consent. To be eligible to participate, subjects were aged 18-65 years. Potential subjects were excluded if they had an existing cardiopulmonary disease and/or neuro(muscular) disease and/or kyphoscoliosis, were unable to understand and read the English or Dutch language, had a history of drug abuse, used respiratory stimulants or depressants or were pregnant.

\section{Study design}

The HCVR was measured using the rebreathing and with the steady-state setup, in two different sessions, 5-9 days apart. The order in which the measurements were performed was randomised, to account for crossover effects on reproducibility over the population. At the second appointment, measurements were performed in the same order, to diminish a change in crossover effect affecting within-subject reproducibility. Subjects were instructed to abstain from caffeine. Subjects were distracted from their breathing activity during the measurement, by watching an episode of a nature documentary.

\section{Methods of measurement}

The HCVR was measured using the rebreathing and steady-state methods. Subjects were connected to a closed system via a Hans Rudolph mask (Shawnee, KS, USA) of appropriate size. The closed system was filled with oxygen (inspiratory oxygen fraction $\left.\left(\mathrm{FIO}_{2}\right)>60 \%\right)$. A y-piece with valves for inspiration and expiration was placed in the system to maintain a one-way circuit. Ventilation was measured using a 
Triple-V flow sensor (Oxycon Pro; Care Fusion, San Diego, CA, USA) and air was sampled to measure breath-by-breath oxygen and $\mathrm{CO}_{2}$ levels. While rebreathing, inspiratory $\mathrm{CO}_{2}$ pressure was continuously raised by exhalation of the subject in the closed system. In steady-state measurement, inspiratory $\mathrm{CO}_{2}$ pressure could be regulated using adjustable valves in a t-piece (two Jaeger (Würzburg, Germany) closing valves in combination with servo motors SG90 $9 \mathrm{~g}$ micro servo (TowerPro Pte Ltd, VYW, Singapore Ardiuno, Somerville, MA, USA) actuated by an Arduino Mega board), partly short-circuiting the $\mathrm{CO}_{2}$ absorber in the expiratory limb of the circuit. Oxygen was continuously added to the closed system, to keep $\mathrm{FIO}_{2}>60 \%$ and suppress peripheral chemoreceptor activity. All measurements were stopped if $\mathrm{PETCO}_{2}$ reached $>8.5 \mathrm{kPa}$, or oxygen saturation $<90 \%$ or if subjects indicated that they would like to stop (e.g. due to feeling too uncomfortable to continue the test).

In both measurements, baseline ventilation was measured $3 \mathrm{~min}$ after stabilisation of minute volume of the subject. In the rebreathing measurement, $\mathrm{PETCO}_{2}$ increased continuously. In the steady-state setup, three distinct levels of $\mathrm{PETCO}_{2}$ were determined (at baseline, at $0.5 \mathrm{kPa}$ above baseline and at $1.5 \mathrm{kPa}$ above baseline). Each level was maintained for $5 \mathrm{~min}$ or longer, until ventilation reached a stable level. Start and end times of the measurement were listed on a worksheet.

After each measurement, three numeric rating scale (NRS) questions were answered on duration, breathing experience and dyspnoea sensation, to assess subjects' experience of the method. At the end of a session, subjects registered which method they preferred on the basis of the three components addressed by the NRS questions and, in addition, an overall preference.

All data were analysed offline using MATLAB 2016a (MathWorks, Natick, MA, USA). All parameters were filtered with a moving average filter of five breaths. Linear regression was used to determine the sensitivity of the chemoreceptors (slope-SS for steady-state and slope-R for rebreathing). In the steady-state measurement, mean $\mathrm{PETCO}_{2}$ and ventilation in the last minute of each level (three points) were used to calculate slope-SS with linear regression (see figure 1 for analysis of example data). In the rebreathing measurement, VRT was determined in the first 10 breaths of the rebreathing phase, using a custom-designed algorithm. $\mathrm{EETCO}_{2}$ data above VRT were used to calculated slope-R with linear regression

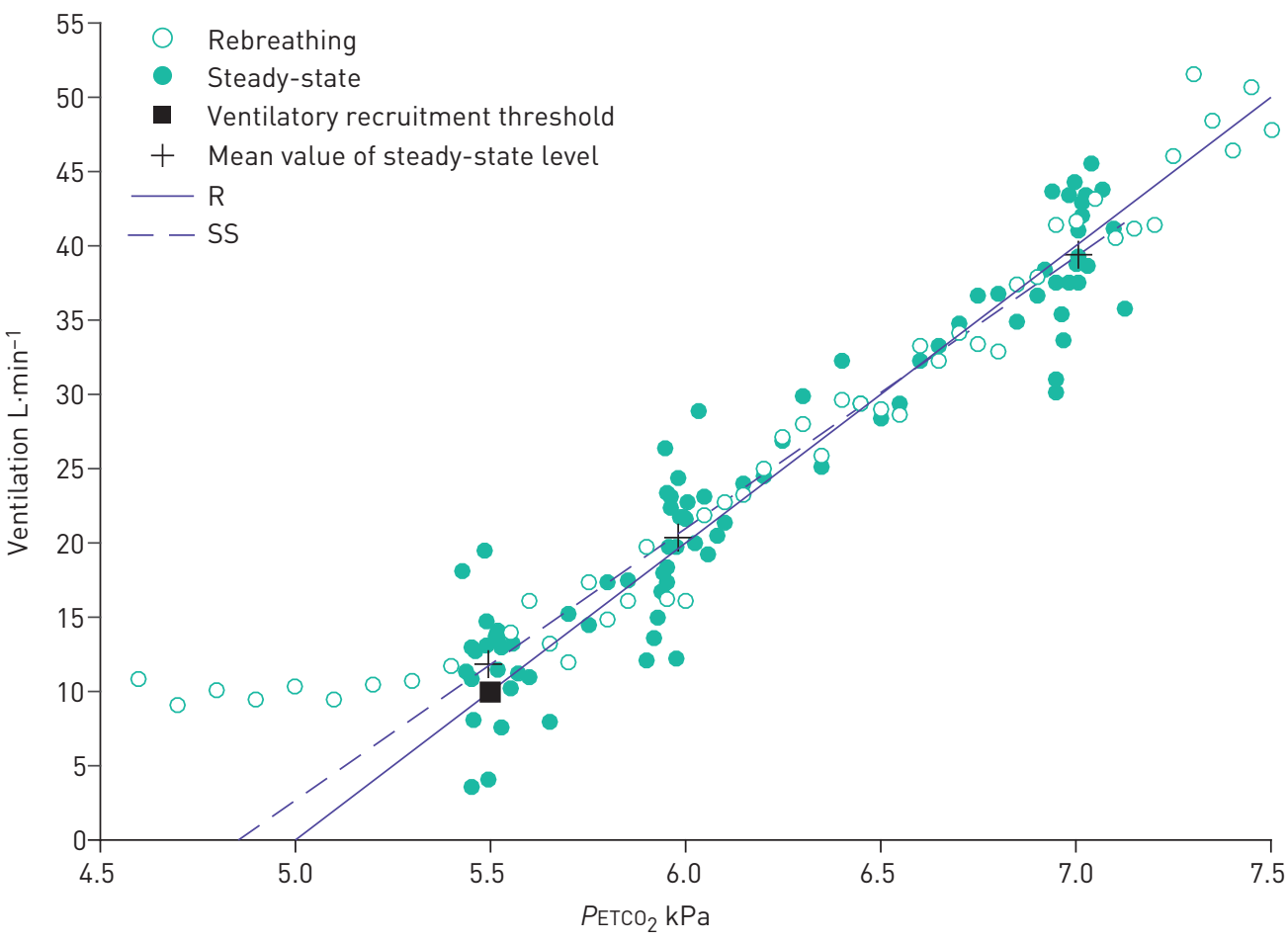

FIGURE 1 Analysis of example data of the hypercapnic ventilatory response. The ventilatory recruitment threshold is used in the rebreathing method to calculate the linear relationship between end-tidal carbon dioxide tension $\left(\mathrm{PETCO}_{2}\right)$ and ventilation (slope R). Three levels can be distinguished in the steady-state data points $(\sim 5.5, \sim 6$ and $\sim 7 \mathrm{kPa})$. The mean $\mathrm{PETCO}_{2}$ and ventilation per level are shown. Linear regression of the three mean $\mathrm{PETCO}_{2}$ and ventilation points gives the sensitivity to carbon dioxide (slope SS). Both the R and SS cross the $\mathrm{x}$-axis; these are the projected apnoea threshold of the rebreathing method and the steady-state method, respectively. 
(figure 1). The total duration of the measurements was documented. The "projected" (as nonexistent in awake subjects) apnoea threshold was calculated (pAT-R and pAT-SS), i.e. the $\mathrm{PETCO}_{2}$ level where, theoretically, minute volume becomes zero (the intercept of the slope-R or slope-SS with the $\mathrm{X}$-axis).

\section{Statistical analysis}

Statistics were performed using IBM SPSS Statistics 24.0 (North Castle, NY, USA). All parameters were tested for normality. All continuous variables were expressed as mean $\pm \mathrm{SD}$ or median (interquartile range (IQR)), as appropriate. Bland-Altman plots were made to assess the differences between the outcomes (slopes and projected apnoea thresholds) in measurement 1 and 2, for both methods. Furthermore, Bland-Altman plots were made to assess the differences between the outcomes in the rebreathing and steady-state method, for both measurements. The limits of agreement were calculated.

An intraclass correlation coefficient (ICC) was calculated to evaluate the correlation between slope and projected apnoea threshold of the first and second measurement. This represents the reproducibility of the methods and is the primary outcome of the study. If the ICC was $>0.8$, the measurements were assumed to be reproducible. A p-value $<0.05$ was considered a significant correlation.

Paired t-tests were performed to find significant differences between the steady-state and rebreathing methods in 1) the outcomes of the questionnaires and 2) duration. A p-value $<0.05$ indicated a significant difference. If data was not normally distributed a Wilcoxon signed rank test was performed.

\section{Results}

26 subjects were assessed for eligibility. Two did not meet the inclusion criteria and two declined to participate. 22 healthy volunteers participated in the study; two subjects were excluded after the first session. One subject was excluded after the first rebreathing measurement, due to persistent complaints of headache and nausea. Another subject withdrew consent after the first visit, citing discomfort. Of the remaining 20 subjects, 11 were male. All obtained parameters were non-normally distributed. The median (IQR) age was 39 (26-53) years, height was $179(169-183) \mathrm{cm}$ and the weight was $72(65-87) \mathrm{kg}$. The results of the slopes and projected apnoea threshold (median and IQR), per method, per measurement of all subjects, can be found in table 1. Two subjects were not able to reach level 3 in the steady-state measurements in the second session, and were excluded in calculations using the slope-SS of measurement 2 .

The ICC $(95 \%$ CI) of slope- $\mathrm{R}$ measurement 1 versus measurement 2 is $0.89(0.73-0.95)(\mathrm{p}<0.01)$. The ICC of slope-SS measurement 1 versus measurement 2 is $0.56(0.14-0.81)(\mathrm{p}=0.01)$. The ICC of pAT-R of measurement 1 versus measurement 2 is $0.84(0.64-0.93)(\mathrm{p}<0.01)$. The ICC of pAT-SS of measurement 1 versus measurement 2 is $0.25(-0.21-0.62)(p=0.14)$. In addition, the ICC was calculated in the two randomised groups (first rebreathing or first steady-state) (table 2).

Two Bland-Altman plots were made of slope-R of measurement 1 versus 2, and of slope-SS of measurement 1 versus 2 (figure 2). Two Bland-Altman plots were made of pAT-R of measurement 1 versus 2, and of PAT-SS of measurement 1 versus 2 (figure 3).

Table 3 presents the NRS scores of measurements 1 and 2. NRS scores were compared between steady-state and rebreathing, using a Wilcoxon signed rank test. Durations of the rebreathing and steady-state tests were not scored significantly differently (p-values 0.62 and 0.94 for measurement 1 and 2 , respectively). Breathing comfort was scored significantly differently between the steady-state and

TABLE 1 Slopes and projected apnoea thresholds for rebreathing (slope-R and pAT-R,

respectively) and steady-state (slope-SS and pAT-SS, respectively) in the first and second

measurement of all subjects



Data are presented as median (interquartile range). " : two subjects excluded from calculations. 
TABLE 2 Intraclass correlation coefficient $(95 \% \mathrm{CI}$ ) of the slope and projected apnoea threshold between measurement 1 and measurement 2 for rebreathing (slope-R and pAT-R, respectively) and steady-state (slope-SS and pAT-SS, respectively), calculated in the two randomised groups

$\mathbf{R}$

$\begin{array}{lc}\text { Slope-R } & 0.90(0.66-0.98) \\ \text { Slope-SS } & 0.49(-0.17-0.84) \\ \text { pAT-R } & 0.90(0.64-0.97) \\ \text { pAT-SS } & -0.08(-0.65-0.55)\end{array}$

$0.87(0.56-0.97)$

$0.58(-0.14-0.90)$

$0.85(0.50-0.96)$

$0.55(-0.80-0.89)$

The outcomes of slope-R and pAT-R were significant ( $p<0.05)$; the outcomes of slope-SS and pAT-SS were not significant $(p>0.05)$.

rebreathing methods ( $\mathrm{p}$-values 0.01 and $<0.01$ for measurement 1 and 2 , respectively). Dyspnoea sensation was significantly different in measurement $1(\mathrm{p}=0.04)$ and almost significant in measurement $2(\mathrm{p}=0.06)$. Breathing was experienced more comfortably in the steady-state measurement, while dyspnoea sensation was less. In both measurements, $16(80 \%)$ out of 20 subjects chose the steady-state as their preferred method, while four chose the rebreathing method. In all three areas (duration, breathing comfort and dyspnoea sensation), the steady-state method was preferred by most subjects (table 4)

The median duration of the steady-state measurements was 23.5 (21-25.8) $\mathrm{min}$ and 22.5 (20-25) $\mathrm{min}$ for the first and second measurements, respectively. The median duration of the rebreathing measurements was 18.5 (17-21.5) min and 17 (15-21) min, respectively, for the first and second measurements. With a Wilcoxon signed rank test a significant difference in duration of the rebreathing tests and the steady-state tests was found $(\mathrm{p}<0.01$ in both measurements). The rebreathing measurements were shorter than the steady-state measurements. The mean difference in duration of measurement 1 was $4.5(2.0-6.8) \mathrm{min}$; for measurement 2 it was $5.5(3.3-6.8) \mathrm{min}$.

\section{Discussion}

The major findings of this study were 1) the rebreathing method is reproducible; 2) the steady-state method is not reproducible; 3 ) steady-state tests are preferred by the subjects; however, absolute differences between the methods seem to be clinically irrelevant; and 4) duration of the rebreathing method was less.
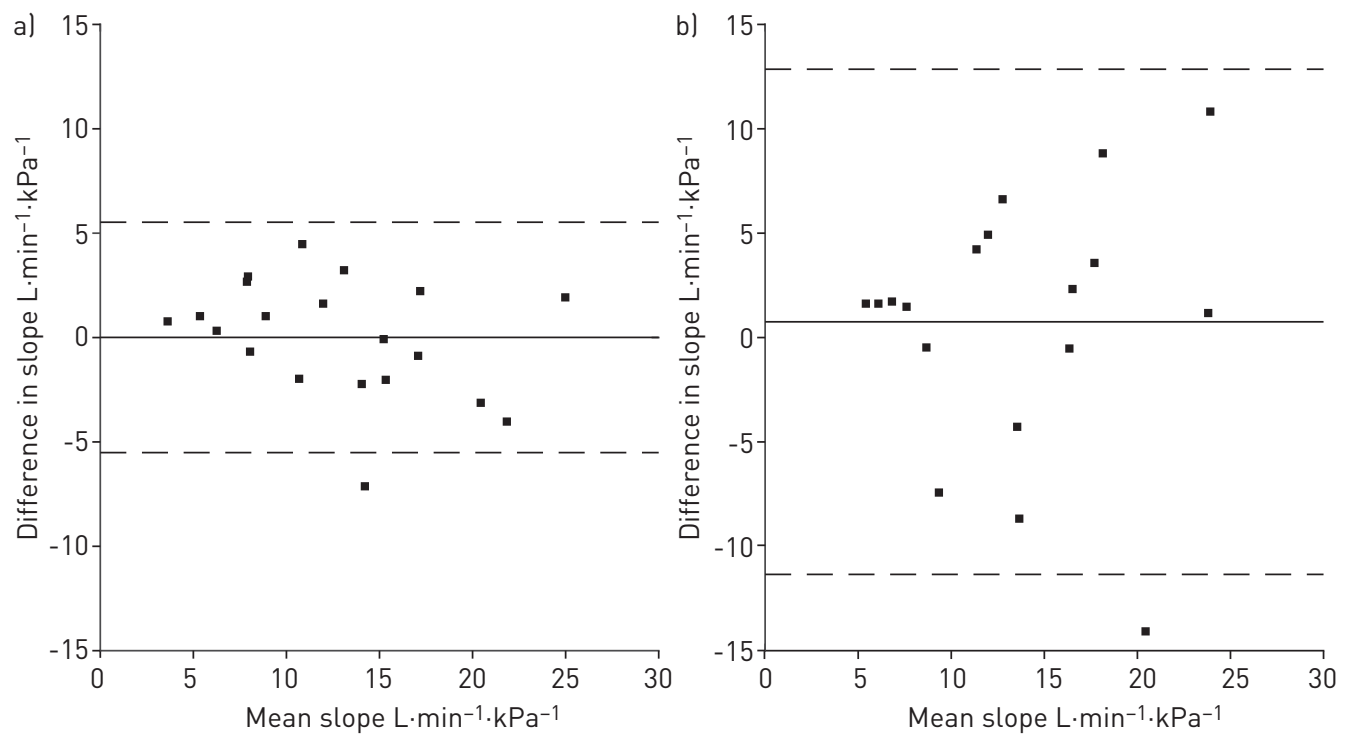

FIGURE 2 Bland-Altman plot of slopes of measurement 1 versus 2. a) Rebreathing method $(n=20)$; b) steady-state method ( $n=18$ ). Mean of slopes on the $x$-axis in $L \cdot \mathrm{min}^{-1} \cdot \mathrm{kPa}{ }^{-1}$ and the difference between slopes on the $y$-axis in $L \cdot \mathrm{min}^{-1} \cdot \mathrm{kPa}^{-1}$. The mean slope difference is for both methods close to zero. The limits of agreement of the rebreathing measurements are $\sim 12 \mathrm{~L} \cdot \mathrm{min}^{-1} \cdot \mathrm{kPa}^{-1}$ and of steady-state measurements are $\sim 26 \mathrm{~L} \cdot \mathrm{min}^{-1} \cdot \mathrm{kPa}^{-1}$. There is no trend in the mean slope. 
a)

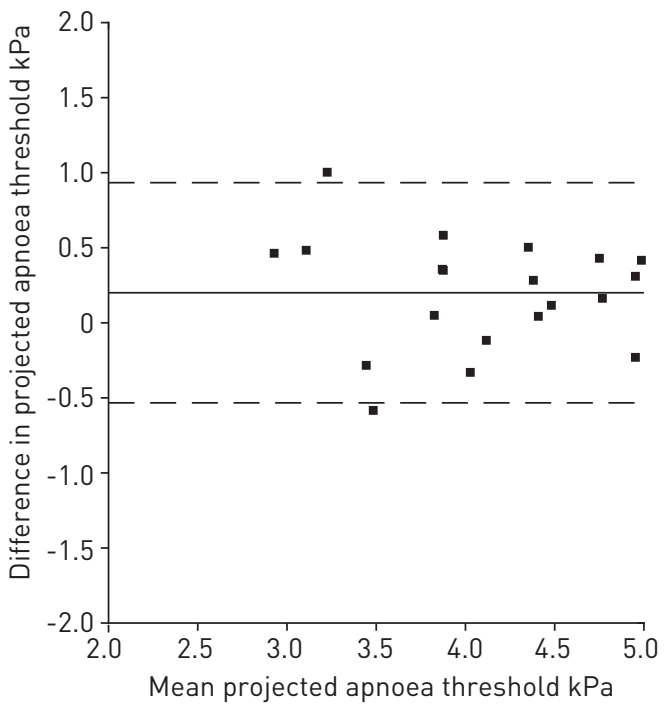

b)

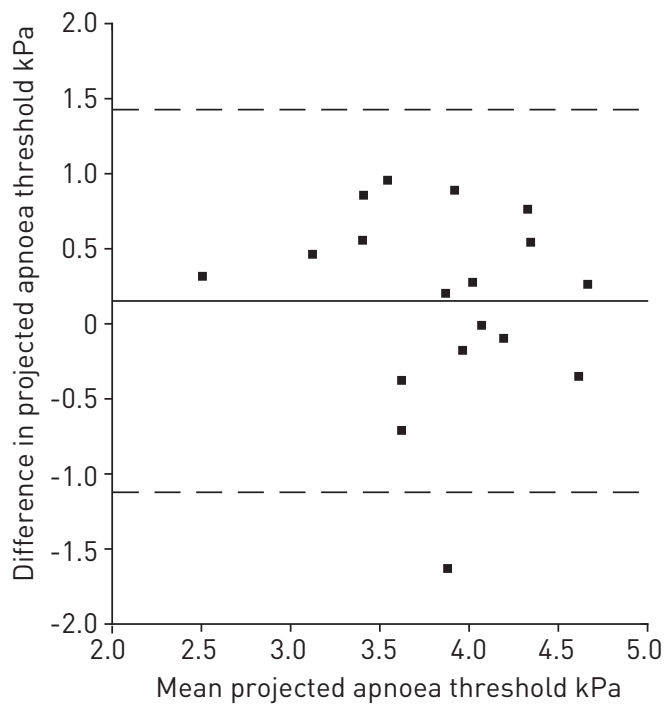

FIGURE 3 Bland-Altman plot of projected apnoea threshold of measurement 1 versus 2. a) Rebreathing $(R)$ method $(n=20)$; b) steady-state (SS) method ( $n=18)$. Mean of projected apnoea threshold (pAT) on the $x$-axis in $\mathrm{L} \cdot \mathrm{min}^{-1} \cdot \mathrm{kPa}^{-1}$ and the difference between pAT on the $\mathrm{y}$-axis in $\mathrm{L} \cdot \mathrm{min}^{-1} \cdot \mathrm{kPa} \mathrm{Pa}^{-1}$. The mean pAT is $\sim 0.3 \mathrm{kPa}$ in both methods. The limits of agreement of pAT-R are $\sim 1.5 \mathrm{kPa}$ and of pAT-SS are $\sim 2.5 \mathrm{kPa}$. There is no trend in the mean projected apnoea threshold.

\section{Reproducibility}

The ICC (95\% CI) of slope-R was $0.89(0.73-0.95)$ and that of pAT-R was $0.84(0.64-0.93)$. The ICC of the steady-state measurements was smaller, i.e. $0.56(0.14-0.81)$ and $0.25(-0.21-0.62)$ for slope-SS and pAT-SS, respectively. The rebreathing measurements are considered reproducible with an ICC $>0.8$, while the steady-state measurements are not. The 95\% confidence interval of the steady-state measurements is wide. In the study by STRACHOVA and Plum [5], the long-term reproducibility of Read's rebreathing method was tested using a Spearman's ranked correlation coefficient in 13 subjects. They found a correlation coefficient of 0.939 between the mean slopes of the first and second sessions. The use of a Spearman's ranked correlation coefficient implicates that a systematic error between measurements is not detected, resulting in a higher correlation. In 2010, JENSEN et al. [8] calculated the ICC of Duffin's rebreathing method under hyper- and hypoxic conditions between days and within days. The ICC of the slopes was $0.78(0.58-0.90)$ and of the apnoeic threshold was $0.82(0.66-0.92)$ between days. The rebreathing protocol of JENSEN et al.'s study differs from the rebreathing protocol used in this study. It is hypothesised that the difference in protocol (addition of hypoxic tests, prior hyperventilation and the use of a prefilled rebreathing bag [4]) explains the small difference in reproducibility in measured slope. The results of the reproducibility of the apnoeic threshold are similar.

Berkenbosch et al. [6] concluded that the slopes of three subjects, measured using a dynamic end-tidal forcing steady-state method, showed good correspondence over consecutive days. This contradicts the

TABLE 3 Numeric rating scale scores on duration (1: very unpleasant, 10: very pleasant), breathing comfort (1: very unpleasant, 10: very pleasant) and dyspnoea sensation (1: not dyspnoeic, 10: very dyspnoeic) for rebreathing and steady-state for the first and second measurements

Measurement 1

Measurement 2

\begin{tabular}{|c|c|c|c|c|}
\hline & & & \\
\hline & Rebreathing & Steady-state & Rebreathing & Steady-state \\
\hline Duration & $6.0(5.0-7.0)$ & $5.5(4.0-7.8)$ & $5.5(5.0-6.8)$ & $6.0(5.0-7.0)$ \\
\hline Breathing comfort & $5.0(4.0-6.0)$ & $6.0(5.0-7.0)$ & $4.5(4.0-5.8)$ & $6.0(4.0-7.0)$ \\
\hline Dyspnoea sensation & $4.0(4.0-5.8)$ & $3.5(2.3-4.8)$ & $4.0(4.0-6.0)$ & $4.0(2.3-5.0)$ \\
\hline
\end{tabular}




\begin{tabular}{|c|c|c|c|c|c|c|}
\hline & \multicolumn{3}{|c|}{ Measurement 1} & \multicolumn{3}{|c|}{ Measurement 2} \\
\hline & Rebreathing & Steady-state & No preference & Rebreathing & Steady-state & No preference \\
\hline Duration & 25 & 50 & 25 & 25 & 55 & 20 \\
\hline Breathing comfort & 20 & 80 & 0 & 20 & 75 & 5 \\
\hline Dyspnoea sensation & 15 & 70 & 15 & 10 & 85 & 5 \\
\hline Overall preference & 20 & 80 & & 20 & 80 & \\
\hline
\end{tabular}

results of the steady-state measurements obtained in this study. If the small population size in the study of Berkenbosch et al. is ignored, several hypotheses can be made for the overall poor reproducibility of steady-state measurements in this study based on differences in study designs, accounting for nonlinearities, sex differences and the steady-state method.

As it is uncertain that nonlinearities $[4,6,8,11,12]$ or sex differences [8, 13-15] play a role in the reproducibility in this study, a post hoc analysis was performed. The slope of the steady-state method was calculated with linear regression of the upper two levels $\left(\mathrm{ETCO}_{2}\right.$ at baseline $+0.5 \mathrm{kPa}$ and $\left.+1.5 \mathrm{kPa}\right)$ and the ICC was determined. The ICC was $0.62(0.23-0.84)(\mathrm{p}<0.01)$; reproducibility increased only slightly when accounting for nonlinearity at level 1 (i.e. level 1 was below VRT). Furthermore, the ICC is calculated separately for males and females. The ICC was $0.51(-0.01-0.84)$ for males and 0.66 $(-0.01-0.93)$ for females. Given these data, it seems unlikely that nonlinearities or inclusion of both males and females have caused the poor reproducibility of our steady-state method.

\section{Subject experience}

No other studies that we are aware of have studied the experience of the subjects with both rebreathing and steady-state tests. 16 out of 20 subjects chose the steady-state measurement as their preferred method at both visits. This can be explained by the greater breathing comfort and lower dyspnoea sensation of the steady-state method. A possible explanation for the greater sensation of dyspnoea of the rebreathing method is that subjects are exposed to higher minute volumes. Furthermore, we observed that side-effects (e.g. nausea, headache) are more present in rebreathing, due to higher $\mathrm{PETCO}_{2}$ values, causing substantial cerebral vasoconstriction and dilation, although this is merely speculation as the side-effects were not listed separately for each test. The absolute difference between NRS scores of both methods were small and seem clinically irrelevant.

\section{Limitations and recommendations}

The steady-state response should be measured at three stable levels of $\mathrm{PETCO}_{2}$, to equilibrate $\mathrm{PETCO}_{2}$ with central $P_{\mathrm{CO}_{2}}$ and tissue $P \mathrm{ETCO}_{2}$. If $P \mathrm{ETCO}_{2}$ is not equilibrated with central $P_{\mathrm{CO}_{2}}$ at one or more levels, the calculation of the slope is influenced. Berkenbosch et al. [6] used the dynamic end-tidal forcing technique to keep $\mathrm{PETCO}_{2}$ levels constant. Therefore, it is certain that an equilibrium between $\mathrm{PETCO}_{2}$ and central $\mathrm{PCO}_{2}$ is reached. To assess the stability of the steady-state measurement in this study, post hoc analysis was performed. $P \mathrm{ETCO}_{2}$ at baseline $+0.5 \mathrm{kPa}$ and $+1.5 \mathrm{kPa}$, showed a mean coefficient of variation of $19 \%$ and $39 \%$, respectively, for measurement 1 and $20 \%$ and 39\%, respectively, for measurement 2. Due to manual positioning of the valves, $\mathrm{PETCO}_{2}$ is prone to fluctuations. Therefore, in this study, it is likely that equilibration between $\mathrm{PETCO}_{2}$ and central $\mathrm{PCO}_{2}$ was insufficient at one or more levels, causing poor reproducibility. Another explanation for the poor reproducibility could be an agitated state of the subjects. However, it is assumed that all subjects were put at ease by watching a nature documentary during the test.

It should be noted that the reproducibility is only tested under hyperoxic conditions and in healthy subjects. This study mainly measured the response of the central chemoreceptors, as hyperoxic conditions were maintained [16]. However, under normal ambient conditions, the peripheral chemoreceptors also affect the measured sensitivity to $\mathrm{CO}_{2}$. The use of hyperoxic conditions decreases the sensitivity to $\mathrm{CO}_{2}$ relative to normoxic conditions $[4,17,18]$. Furthermore, the HCVR in patients is altered relative to the HCVR of healthy subjects. It is possible that the reproducibility of the measurements changes when normoxic conditions are applied or when patients are measured. Reassessment of the reproducibility is recommended in these two situations. 


\section{Summary}

Measurement of the hypercapnic ventilatory response using the rebreathing setup provided reproducible results, while the steady-state setup did not. Poor reproducibility of the steady-state method may be explained by high variability in end-tidal $\mathrm{PCO}_{2}$. Differences in subject experience between the methods are small.

\section{Acknowledgements}

The authors would like to thank Marcel Vlutters and Hans Nieland (Pulmonary Function Dept, Medisch Spectrum Twente, Eschende, the Netherlands), for their help with the construction of the measurement setup and Job van der Palen (Medical School Twente, Medisch Spectrum Twente), for assistance in the design of the study.

\section{References}

Prabhakar NR. Oxygen sensing by the carotid body chemoreceptors. J Appl Physiol 2000; 88: 2287-2295. Duffin J. Role of acid-base balance in the chemoreflex control of breathing. J Appl Physiol 2005; 99: 2255-2265. Mohan R, Duffin J. The effect of hypoxia on the ventilatory response to carbon dioxide in man. Respir Physiol 1997; 108: 101-115.

4 Duffin J. Measuring the respiratory chemoreflexes in humans. Respir Physiol Neurobiol 2011; 177: 71-79.

5 Strachova Z, Plum F. Reproducibility of the rebreathing carbon dioxide response test using an improved method. Am Rev Respir Dis 1973; 107: 864-869.

6 Berkenbosch A, Bovill JG, Dahan A, et al. The ventilatory $\mathrm{CO}_{2}$ sensitivities from Read's rebreathing method and the steady-state method are not equal in man. J Physiol 1989; 411: 367-377.

7 Cohen G, Henderson-Smart DJ. The reproducibility of the response of the human newborn to $\mathrm{CO}_{2}$ measured by rebreathing and steady-state methods. J Physiol 1994; 476: 355-363.

8 Jensen D, Mask G, Tschakovsky ME. Variability of the ventilatory response to Duffin's modified hyperoxic and hypoxic rebreathing procedure in healthy awake humans. Respir Physiol Neurobiol 2010; 170: 185-197.

9 MacKay CM, Skow RJ, Tymko MM, et al. Central respiratory chemosensitivity and cerebrovascular $\mathrm{CO}_{2}$ reactivity: a rebreathing demonstration illustrating integrative human physiology. Adv Physiol Educ 2016; 40: 79-92.

10 Price HL. Effects of carbon dioxide on the cardiovascular system. Anesthesiology 1960; 21: 652-663.

11 Mohan RM, Amara CE, Cunningham DA, et al. Measuring central-chemoreflex sensitivity in man: rebreathing and steady-state methods compared. Respir Physiol 1999; 115: 23-33.

12 Pandit JJ, Mohan RM, Paterson ND, et al. Cerebral blood flow sensitivity to $\mathrm{CO}_{2}$ measured with steady-state and Read's rebreathing methods. Respir Physiol Neurobiol 2003; 137: 1-10.

13 Beidleman BA, Rock PB, Muza SR, et al. Exercise $V^{\prime} \mathrm{E}$ and physical performance at altitude are not affected by menstrual cycle phase. J Appl Physiol 1999; 86: 1519-1526.

14 MacNutt MJ, De Souza MJ, Tomczak SE, et al. Resting and exercise ventilatory chemosensitivity across the menstrual cycle. J Appl Physiol 2012; 112: 737-747.

15 Jensen D, Webb KA, O'Donnell DE. Chemical and mechanical adaptations of the respiratory system at rest and during exercise in human pregnancy. Appl Physiol Nutr Metab 2007; 32: 1239-1250.

16 Dempsey JA, Veasey SC, Morgan BJ, et al. Pathophysiology of sleep apnea. Physiol Rev 2010; 90: 47-112.

17 Dahan A, DeGoede J, Berkenbosch A, et al. The influence of oxygen on the ventilatory response to carbon dioxide in man. J Physiol 1990; 428: 485-499.

18 Duffin J, McAvoy GV. The peripheral-chemoreceptor threshold to carbon dioxide in man. J Physiol 1988; 406: $15-26$. 